\title{
Der morphologische Aspekt der Kontamination im Deutschen und im Arabischen, eine vergleichende Studie
}

Vorgelegt von

Taghrid Ibrahim Abdelraouf Mohamed

(Masterandin)

Betreut von:

Ass. Prof. Dr. Shukry Muhammad Abdelbaky

Ass. Prof. für Linguistik an der Abteilung für Germanistik der Sprachenund Übersetzungsfakultät der Al-Azhar Universität, Kairo

Ass. Prof. Dr. Marwa Abdelmohsen Osman Ziko

Ass. Prof. für Linguistik an der Abteilung für Germanistik der

Fakultät der geisteswissenschaftlichen Studien der Al-Azhar

Universität- Frauenzweig, Kairo

The morphological aspect of contamination in Arabic and

German, a comparative study

Abstract: The research deals with dealing with the morphological aspect of contamination in the Arabic and German language through the comparative study. The research aims to study contamination forms from the morphological side in both languages. It also aims to study the types of word that form the contaminate in both languages. In addition to dealing with the morphological structure of contamination through models describing the process of building and forming a contaminate in the German language. The research deals with the study of the two most important models in dealing with the morphological side in German. The research aims to presenting the rules that linguists have devised to build contaminate.

The results of the research indicated that contamination is one of the linguistic phenomena in both languages, which have morphological characteristics that show some differences and similarities in both languages. Contamination builds in both languages by combining at least two words into one word or by

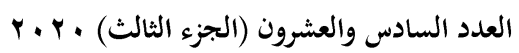

(319)

مجلة كلية التربية- جامعة عين شمس 
combining two words in one word, in order to form a new word. Contamination words come as names, verbs or adjectives in both languages. Relative contamination comes in Arabic and is not found in German. Other types of word types also appear in German. We find contaminations of numbers, contaminations of conditions, contaminations of pronouns, and others.

Key words: contamination, word classes, morphology, contamination forms, models, rules. 


\section{Taghrid Ibrahim Abdelraouf Mohamed}

\section{الجاتب الصرفى للنحت فى اللغة العربية والألمانية، دراسة مقارنة}

الملخص: يتتاول البحث معالجة الجانب الصرفى للنحت فى اللغة العربية والألمانية من

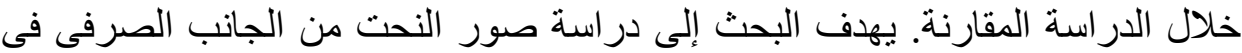

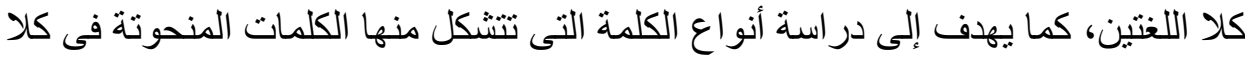

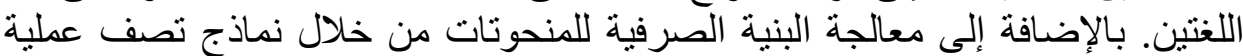

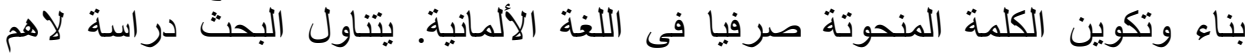

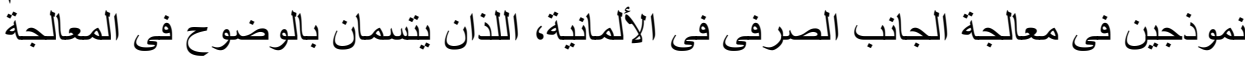

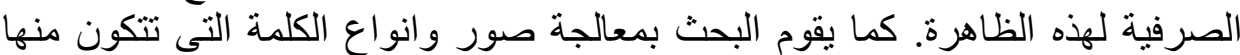

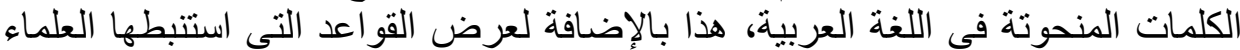

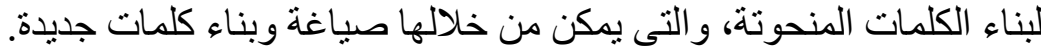

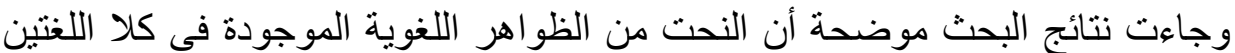

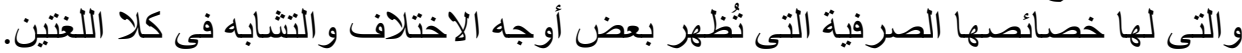

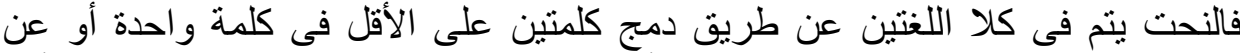

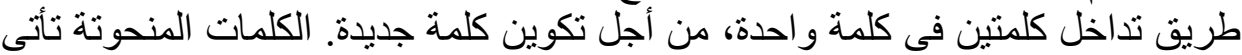

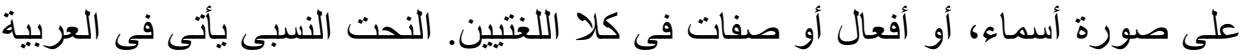

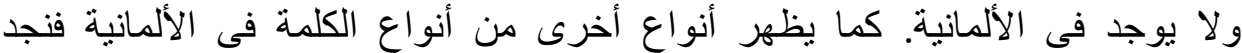

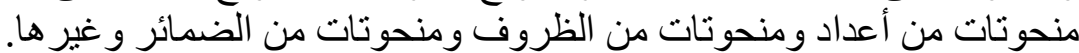

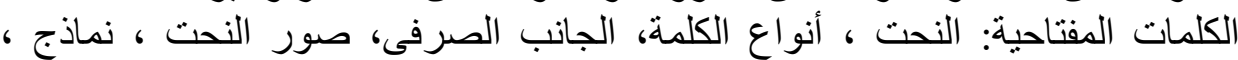

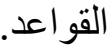




\title{
Der morphologische Aspekt der Kontamination im Deutschen und im Arabischen, eine vergleichende Studie
}

\author{
Vorgelegt von \\ Taghrid Ibrahim Abdelraouf Mohamed \\ (Masterandin) \\ Betreut von:
}

Ass. Prof. Dr. Shukry Muhammad Abdelbaky

Ass. Prof. für Linguistik an der Abteilung für Germanistik der Sprachenund Übersetzungsfakultät der Al-Azhar Universität, Kairo

Ass. Prof. Dr. Marwa Abdelmohsen Osman Ziko

Ass. Prof. für Linguistik an der Abteilung für Germanistik der

Fakultät der geisteswissenschaftlichen Studien der Al-Azhar

Universität- Frauenzweig, Kairo

\section{Einleitung}

Das Phänomen der Kontamination ist ein umstrittenes Phänomen, das in allen Sprachen vorhanden sind. Dieses Phänomen kommt im Deutschen und im Arabischen vor, wobei die Kontamination durch die Verschmelzung oder Überlappung mindestens zweier Wörter zu einem neuen Wort entsteht. Aber jede Sprache hat ihre morphologischen Besonderheiten bei der Bildung von Kontamination. Die vorliegende Arbeit beschäftigt sich mit der kontrastiven Untersuchung der Kontamination im Hinblick auf den morphologischen Aspekt im Deutschen und Arabischen.

\subsection{Problemstellung und Ziel der Arbeit}

Die vorliegende Arbeit behandelt den morphologischen Aspekt der Kontaminationsformen in der deutschen und arabischen Wortbildung. Diese Arbeit hat die kontrastive Untersuchung des morphologischen Aspekts der Kontamination in beiden Sprachen als Ziel. Im Rahmen der Arbeit werde ich auf folgende Fragen zu antworten versuchen:

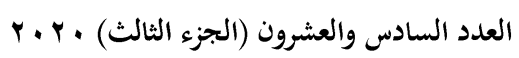

(322)

مجلة كلية التربية- جامعة عين شمس 
Taghrid Ibrahim Abdelraouf Mohamed

1. Gibt es im Deutschen und Arabischen bestimmte Regel bei der Bildung von Kontamination?

2. Welche Formen der Kontamination kommen im Deuschen vor?

3. Welche Formen der Kontamination kommen im Arabischen vor?

4. Gibt es Gemeinsamkeiten und Unterschiede der vorhandenen Formen von Kontamination in beiden Sprachen?

5. Wie kann man die Kontamination morphologisch beschreiben?

\subsection{Methode der Arbeit}

In der vorliegenden Arbeit wird die deskriptiv vergleichende Methode verwendet, um das Phänomen "Kontamination" hinsichtlich des morphologischen Aspekts im Deutschen und Arabischen $\mathrm{zu}$ behandeln und $\mathrm{zu}$ beschreiben.

\subsection{Forschungsstand der Arbeit}

Die morphologische Beschreibung der Kontamination wird von den meisten Sprachwissenschaftlern im Deutschen und im Arabischen untersucht und behandelt. Darunter sind im Arabischen: Āmyn (2000), Ālmaǵraby (1908), Tarzy (2005), Ḡirğis (1961), Ālsawahily (1994), 'ukašah (2002), Ālqinīby (1992) u.a.; im Deutschen sind Friedrich (2008), Kessel/ Reimann (2008), Ronneberger-Sibold (2005) Bußmann (2002), Lohde (2006), , Donalies (2011) u. a.

\subsection{Gegenstand und Aufgliederung der Arbeit}

Diese Arbeit behandelt den morphologischen Aspekt der Kontamination im Deutschen und Arabischen. Diese Arbeit gliedert sich in Einleitung, zwei Kapitel und Literaturverzeichnis. Im ersten Kapitel werden die Wortklassen der Kontamination im Deutschen, die morphologische Beschreibung des 
Kontaminationsprozesses im Deutschen, Bußmanns Modell zur morphologischen Beschreibung und RonnebergerSibolds Modell auf der Grundlage der relativen Transparenz dargestellt. Im zweiten Kapitel werden die Formen der Kontamination im Arabischen, die Wortklassen der Kontamination im Arabischen, die morphologischen Regel bei der Bildung von Kontamination und die Schlussfolgerung durchgeführt.

\section{Morphologische Beschreibung der Kontamination im Deutschen}

\subsection{Wortklasse der Kontamination im Deutschen}

Die meisten Sprachwissenschaftler sind der Ansicht, dass die Kontamination bei den Substantiven produktiv ist: Friedrich (2008) z. B. stützt sich bei ihrer Dissertation auf die Belege, die beweisen, dass die Kontamination bei den Substantive produktiv ist. Diese Belege enthalten ungefähr $86 \%$ Substantive, $7 \%$ Adjektive und 5 $\%$ Verben. Zudem lassen sich einige weiteren Wortarten entdecken, die rund $2 \%$ beanspruchen. Als Beispiele für das Auftreten weiterer Wortarten sind: Die Antwortpartikeln jein, PORNO, Olymp-ja!; die Interjektionen Juch-Hai!, E-GITT; die Gesprächspartikeln Bon(n)jour, Kaufwiedersehen! Insgesamt sind 14 Partikeln als Belege. Außerdem sind die sechs Adverbien: „Nachhair, SITENWEISE, EXTRA, allerhandy, SOSehr und WARum? Auch noch ,,die vier Numeralia drölf, 04i,19990ethe. Hinsichtlich der Pronomina sind die zwei Indefinit-Pronomen man(n) und Mee(h)r. Es gibt nur einen indefiniten Artikel Eierlei (vgl. Friedrich 2008: 151f.). Friedrich (2008: 153) konstatiert, dass es keine Beschränkung bezüglich der Wortarten der Ausgangseinheiten gibt. Daher könnten Basiselemente aller Wortarten vorkommen. Ausnahmen findet sie bei Konjunktionen. Die Ausgangseinheiten sind Kontaminationen unterschiedlicher Wortbildungstypen, so finden sich Simplizia (drölf), Komposita wie (Mausratsversicherung), Derivate wie (Mauszubildender), 
Kurzwörter (e-conomy, MiTBwohner) und Kontaminationen wie (Wellance) als Basiselemente. Außerdem fügte sie Konversionen hinzu. Z.B. bei den Substantiven Ja und Nein zu dem Wort Jein. Diese Bildung stellt sie neben der Partikel jein dar. Die Produktivitätsmöglichkeit der Kontaminationen zeigen sich vor allem bei den Substantiven und Adjektiven. Als Beispiel dafür nennt sie: Ostalgie (gebildet aus den Substantiven: Osten und Nostalgie), modro (gebildet aus den Adjektiven: modern und retro in einer Werbung für Nissan) (vgl. Kessel/ Reimann 2008: 117). Lohde (2006: 44) hebt hervor, dass die Kontaminationen an der ersten Stelle bei Substantiven vorkommen, aber seltner bei den Adjektiven und Verben auftreten. Er führt einige Beispiele als Belege für seine Auffassung und für ihre Produktivität in der Werbund Pressesprache an: Z. B. Milka aus Milch und Kakao, Nescafé aus Nestlé und Café. Nach Donalies (2011: 71) werden sich bei der Bildung von Kontamination zwei Substantiv-Substantiv-Komposita überlappt wie Hefezopfspange. Mitunter können die Kontaminationen aus zwei Adjektiven gebildet werden, wie akadämlich aus den beiden Adjektiven akademisch und dämlich. Manchmal kann man die Kontaminationen aus zwei Verben prägen, wie Philosofaseln aus Philosophieren und faseln. Seltner sind Bildungen aus verschiednen Wortarten wie Utopiate aus utopische Opiate, Teuro aus teuer und Euro oder computent aus Computer und kompetent. Kontaminate, die aus Lexemen gleicher Wortart gebildet werden, haben eine kopulative Lesart: z. B. akadämlich ist sowohl akademisch als auch dämlich. Aber Kontaminate aus Wörtern verschiedener Wortarten haben nur determinative Lesart: z. B. fahradiesisch ist paradiesisch fürs Fahrrad (vgl. Donalies 2011: 71). Im folgenden werden die morphologischen Beschreibungen der Kontamination dargestellt, um dieses Phänomen präziser zu verstehen: 


\subsection{Morphologische Beschreibung des Kontaminationsprozesses im Deutschen}

Bei der Behandlung des morphologischen Aspekts konzentriere ich mich auf die Modelle, die unterschiedlich in ihren Klassifizierungen erscheinen und die sich in ihren Ergebnissen ergänzen, um dieses Phänomen zu analysieren:

\subsection{Bußmanns Modell zur morphologischen Beschreibung:}

Bußmann unterscheidet zwischen unwillkürlicher Kontamination, die aus Fehlerprägungen entstehen, und absichtlichen Prägungen von Kontaminationen: Die unabsichtliche Kontamination -wie z.B. Opernstecher, gebildet aus: Operngucker und Feldstecher- hat keine Regel für die Verschmelzung (vgl. Bußmann 2002: 373). Bußmann (2002: 373) stellt für die absichtlichen Kontaminationen, die aus stilistischen Gründen gebildet werden, fünf Typen von Kontamination fest:

1. „Haplologische“" vereinfachende Zusammenziehungen, bei denen die Überschneidung der beteiligten Einheiten vorkommt, wenn der erste Teil des ersten Wortes mit dem ersten Teil des zweiten Wortes das gleiche Segment hat. Z. B. dt. Katzenjammertal, Berlinienbus, gebildet aus: Katzen Jammer + Jammertal, Berlin+ Linienbus. Bei einigen von haplologischen Zusammen- ziehungen liegen aber nur die Überschneidungen von Laut- und Silbeneinheiten vor, wenn sie gemeinsames Laut oder Segment hat. Z.B. tragikomisch gebildet aus: tragisch + komisch.

2. „Wortsplittern', bei denen zwei verschiedene Wörter gekreuzt werden und dadurch Neubildungen entstehen. Z. B. Demokratur, Medizyniker, gebildet aus Demokratie + Diktatur; Medizin + Zyniker.

3. ,analogische Bildungen", die dadurch entstehen, dass ein ähnlich klingendes Lexem anstelle von dem Grundwort gesetzt wird. Z. B. Millionarr, Phrasenmäher, analogisch zu Millionär; Rasenmäher. 
4. „, orthographische Variante ${ }^{6 /}$, die nur durch die Orthographie als Kontaminationen erkennbar sind. Z. B. Schlawiene, gebildet aus: Schlawiner und Wiener.

5. „,Subtraktion“", bei denen ein Laut des Grundwortes zur Bildung eines neuen Morphems, substrahiert wird, z. B. Ostalgie, gebildet aus: Osten und Nostalgie (vgl. Bußmann 2002: 373f.). Bußmann (2002) führt die ,analogischen Bildungen“" an, die die meisten Sprachwissenschaftler vernachlässigen. Bei diesen Bildungen kann das Problem der Zuordnung der Kontamination zu den analogischen Bildungen oder $\mathrm{zu}$ den haplologischen vereinfachenden Zusammenziehungen auftreten: Die Bildung Phrasenmäher kann in Bezug auf die Analogiebildung Rasenmäher als analogische Bildung analysiert werden, trotzdem kann man sie als haplologische vereinfachende Bildung wegen der lautlichen Überschneidung von Phrasen und Rasenmäher bezeichnen, weil die beiden Ausgangswörter über ein gemeinsames Segment verfügen.Wertvoll finde ich ihre Benennungen der Kontaminationsprodukte, bei denen sie den Vorgang und den Prozess der Kontamination zu beschreiben versucht.

Bußmann beschreibt aber dieses Phänomen etwa undifferenziert: Sie vernachlässigt die Behandlung der Wortarten der Kontaminationsprodukte. Bei Bußmann findet sich keine klare begriffliche Abgrenzung der zwei Haupttypen von Kontamination: Wortkreuzung und Wortüberschneidung.

\subsubsection{Ronneberger-Sibolds Modell "Auf der Grundlage der relativen Transparenz':}

Ronneberger-Sibold (2005: 205-223) verwendet die Bezeichnung Wortkreuzung bei der Behandlung der Kontamination. Darunter versteht sie ,,absichtlich durchgeführte Zusammenfügungen von zwei oder selten mehr existierenden Wörtern zu einem neuen in einer Art und Weise, die nicht den Regeln oder Mustern der deutschen Komposition entspricht" (Ronneberger-Sibold 2005: 207f.). Aus der oben genannten Definition versucht sie, die Kontamination von der regulären Wortbildung vor allem der 


Komposition und von den extragrammatischen

Wortschöpfungsverfahren in ihrem Beitrag abzugrenzen. Aber ich beschränke mich bei der Darstellung ihres Modells nur auf ihre Klassifizierung von Kontaminationen in Bezug auf die Transparenzgrade der Kontaminationen. Ronneberger-Sibolds Modell (2005: 206) geht von der Behauptung aus, dass es bei Kontaminationen um individuelle, extragrammatische Wortschöpfungen geht, die bewusst von der Norm abweichend gebildet werden, um einen bestimmten kommunikativen Zweck zu erfüllen. Außerdem diskutiert sie in ihrem Beitrag die Frage, ob die Sprachbenutzer in der Lage sind, verschiedene Transparenzgrade der Bildungen mit Verbindung ihrer Funktionalität für bestimmte kommunikative Zwecke wahrzunehmen (vgl. Ronneberger-Sibold 2005: 206). Bei der Kontamination betrachtet sie, dass die Kontamination im Vergleich zur regulären Komposition hingegen immer reduziert ist. Die Reduktion verändert sich ,,je nach Input und eingesetzter Kreuzungstechnik“". Sie stellt in ihrem Aufsatz diese Techniken dar, die ihre Grundbausteine, die Kürzung und Überlappungen von Bestandteilen sind. Als Beispiel für die Kürzung der Bestandteilen ist Cujasuma aus Cuba $\times$ Java $\times$ Sumatra. Für die Überlappung erwähnt sie Kamelefant aus Kamel $\times$ Elefant, indem sich die gemeinsamen Konstituenten überschneiden (Ronneberger-Sibold 2005: 209). Im Hinblick auf das Beispiel der Kürzung, also „Cujasuma“" kann das Abgrenzungsproblem zwischen der Kontamination und der regulären Kürzung auftreten, wobei die Kürzung durch die Anfangsbuchstaben jeder beteiligten Ausgangswörter gebildet sind. Ronneberger-Sibold (2005: 209) konstatiert, dass die Reduktion der Transparenz in Kontamination verschiedene Zwecke erfüllt: Erstens kann ein starkes formales Verschmelzen der Bestandteile ein Abbild des Verhältnisses zwischen deren Referenten. Das heißt: Es ist ikonisch motiviert. Ein typisches Beispiel ist Smog gebildet aus smok $\times$ fog, das auf Nebel und Rauch, die unauflösbar ineinander übergehen. Zweitens lassen sich die Kontaminationen aus spielerischen Gründen bilden, um

r. r. • العدد السادس والعشرون (الجزء الثالث)

(328)

مجلة كلية التربية- جامعة عين شمس 
dem Rezipienten die Freude einer Verrätselung des Ausdrucks zu machen. Das ist etwa in satirischer Literatur. Drittens führt der Verzicht auf die Transparenz der Bildung zu größeren Freiheit in ihrer lautlichen Gestaltung. Das erklärt sie anhand vom Beispiel Cujasuma. Der Cujasuma nachgebildete Name „Subrajama“, aus Sumatra $\times$ Brasilien $\times$ Java, zeigt die individuelle Freiheit bei der Verwendung von Wortschöpfungstechnicken. Das ist hier dieser Kreuzungstechnik ungewöhnlich, aber nicht ungrammatisch, weil keine Regel vorliegen, nach denen ein Vorgehen bei der Wortschöpfung ,richtig“" oder ,falsch“" ist (vgl. RonnebergerSibold2005:209f). Ronneberger-Sibold (2005: 213-219) Skala der Transparenzgrade in Kontamination ist im Folgenden untergliedert:

1. „Vollkreuzungen“", die sich in den beiden folgenden Typen gegliedert sind:

\section{1 ,Teleskopkreuzung “' 1.2 ,Einschlusskreuzung, 2.} „Konturkreuzung ${ }^{66}$,

\section{3. „Halbvollkreuzung6", 4. ,Splitterkreuzung ${ }^{66}$}

1. Der erste Typ „Vollkreuzungen“" ist dadurch gekennzeichnet, dass seine Bestandteile voll in der Kreuzung erhalten sind und das führt dazu, dass sie leicht $\mathrm{zu}$ erkennen sind. Fast alle Vollkreuzungen gehören zu den Teleskopkreuzungen oder zu den Einschlusskreuzungen (vgl. Ronneberger-Sibold 2005: 213f.).

1.1 Teleskopkreuzungen: Ronneberger-Sibold (2005: 213) stellt fest, dass bei den Teleskopkreuzungen die Bestandteile der Wörter, die sich an den Kreuzungen beteiligt sind, nebeneinanderstehen werden, in dem die Verbindung des Endes des ersten Bestandteils mit dem Anfang des zweiten überlappt. Bei solchen Kreuzungen unterscheidet Ronneberger-Sibold (2005: 213f.) zwischen drei Grade der Transparenz folgendermaßen: Besonders deutlich erkennbar sind solche Kreuzungen, wenn das Gelenk (das Ende des ersten Bestandteils mit dem Anfang des zweiten) ein gemeinsames Morph darstellt wie „Schimmel“" in der Bildung Amtsschimmelpilz gebildet aus: Amtsschimmel $\times$ Schimmelpilz. Die Bestandteile der Kreuzung sind weniger transparent, wenn das Gelenk hingegen

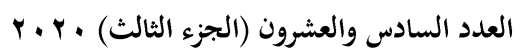

(329) 
durch eine willkürlich ausgewählte Laut- und Buchstabenfolge gebildet wird. So ist der Fall in „Agitproperette“" aus: Agitprop $\times$ Operette und in ," Kamelefant" aus: Kamel $\times$ Elefant. Die Transparenz ist noch etwa geringer, wenn die Kreuzung durch die Überlappung des gemeinsamen Lautes nicht durch Buchstabenfolge gebildet wird. Das ist wie in ,Bilanzknecht" aus: Bilanz $\times$ Landsknecht oder „Kokainszeichen“ " aus: Kokain $\times$ Kainszeichen (vgl. Ronneberger-Sibold 2005: 213f.).

1.2 Einschlusskreuzungen: Bei den Einschlusskreuzungen schließt ein Bestandteil den (oder selten die) anderen Bestandteile als einen Teil seiner Lautfolge in sich ein. Die Struktur dieser Bildung ist nur transparent durch die Orthographie. Das ist der Fall bei „alternativ“", das die Lautfolge /ti:f/ enthält, die sich auf das Wort „tief“" beziehen lässt, sodass die Einschlusskreuzung „, alternatief“ “ gebildet werden lässt (vgl. Ronneberger-Sibold 2005: 214). In einer Einschlusskreuzung bemerkt Ronneberger-Sibold (2005: 215), dass „das einschließende Wort mehr formales Gewicht als das eingeschlossene " zeigt. Aus diesem Grund ist es für den Analyseprozess primär. Das hält sie auch für die folgende Großgruppe der Kontamination „Konturkreuzungen“ “

2. Konturkreuzungen: Bei einer Konturkreuzung wie etwa Tomoffel, Müllionärin, Konkurz ist ,, das formal gewichtigere Wort", also Kartoffel in Tomoffel; Millionärin in Müllionärin; Konkurs in Konkurz, das Ronneberger-Sibold (2005: 215) als Matrixwort bezeichnet, nicht voll in der Kreuzung vorhanden ist. Aber es umhüllt das eingekreuzte Wort, also Müll, Tomat, Konkurz, durch eine für seine Wiedererkennung sehr wichtige Eigenschaft, und zwar seine rhythmische Gesamtkontur. Es definiert durch die Silbenzahl und Akzentsitz, ,,also - '- - von Kartoffel in Tomoffel, -'-- von Millionärin in Müllionärin und - '- von Konkurs in Konkurz" .

Ronneberger-Sibold (2005: 215f.) beschreibt die segmentale Ebene der Konturkurzung, in dem ,das Matrixwort bis auf ganz, ganz 
wenige Ausnahmen den Tonvokal, also das /o/ von Tomoffel, das /ä/ von Müllionärin und das /u/ von Konkurz" c zur Verfügung steht. Sie erläutert, dass jedes Wort aus zwei Teilen besteht: Die Nachkontur und die Vorkontur. Der Tonvokal bildt mit allen folgenden Segmenten die Nachkontur, die in der Poesie als Endreim bekannt ist, also ,,-offel“", ,-ärin“", ,,-urs“" und der ganze Teil des Ausdrucks vor dem Tonvokal bildt die Vorkontur. Das hält sie für eine sehr wichtige Grenze für das Funktionieren der Konturkreuzungen, in dem das zweite Wort entweder in die Voroder die Nachkontur des Matrixwortes eingekreuzt sein kann, wobei die Grenze außer bei Überlappung nicht überschritten werden darf (vgl. Ronneberger-Sibold 2005: 216). Typisch ist die Verwendung des Anfangs des eingekreuzten Wortes anstelle vom Beginn des Matrixwortes. Diese Einkreuzung darf maximal die ganze Vorkontur ausfüllen wie in „Tomoffel“"(- Matrixwort ist: Kartoffel,Vorkuntur ist: Kart-). Bei vielen Bildungen wird dieser Spielraum jedoch gar nicht ausgenutzt, wenn das eingekreuzte Wort wie bei Müllionärin zu knapp ist, wobei der Rest der Vorkontur vom Matrixwort ausgefüllt wird. Ronnberger-Sibold (2005: 216) bemerkt, dass vor allem der Anlaut der Tonsilbe oft vom Matrixwort übernommen wird. Sie behauptet, dass die Überlappungen zwischen Matrixwort und eingekreuztem Wort wie bei Müllionärin die Transparenz steigern. Nach Ronneberger-Sibold (2005: 216) kommen die Einkreuzungen in die Nachkontur des Matrixwortes seltener vor, wie bei Konkurz < Konkurs x kurz. Aus der Analyse dieses Beispiels findet sie, dass die Tonvokale und eventuell sogar Teile der Vorkontur überlappt. Deshalb ist in diesem Fall das eingekreuzte Wort nur nach dem Tonvokal ,sichtbar-bzw. hörbar". Es existiert einige außerordentlichen seltenen Fälle, in denen ein eingekreuztes Wort auch den Tonvokal verändert, als Beispiel ist Heinrich Heins aristokrätzig. Ronneberger-Sibold (2005: 217, Anm.14) erklärt, dass sie die Termini Vor- und Nachkontur von Hockett (1967: 914) und Dressler (1976), die sie für historische und versehentliche Prägungen verwendeten,

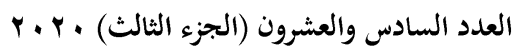


übernahm. Sie verweist darauf, dass Cannon (1986: 746) und Rainer (1993: 88) bemerkten, dass die Silbenzahl und der Akzentsitz von Kontaminationen oft durch das Längere der Ausgangswörter bestimmt sind. Ronneberger-Sibold (2005: 217) fügt hinzu, dass das Matrixwort oft aber nicht immer länger als das eingekreuzte Wort ist. Der ,springende Punkt“, den sie aus der vorgelegten Analyse herausstellt, ist die ,gestalthafte Zusammenschau der verschiedenen Faktoren“. Das Matrixwort ist an seiner rhythmischen Gestalt in Verbindung mit seinemTonvokal und typischerweise dem Rest der Nachkontur eindeutig. Aufgrund der Transparenz des Matrixwortes wird ein semantischer ,frame“"eröffnet, und kann man innerhalb von diesem das eingekreuzte Wort an seiner bloßen Vorkontur erkennen, sofern es nicht ohnehin komplett in der Kreuzung vorhanden ist. Aus ihrer Analyse erschließt Ronneberger-Sibold, dass für das Erkennen der Elemente einer Wortkreuzung deren Distribution von größerer Bedeutung ist als deren Länge. Sie erläutert mit diesem Satz ihre Folgerung: „Es müssen nicht unbedingt möglichst viele Segmente aus den $\mathrm{zu}$ kreuzenden Wörtern erhalten bleiben, sondern die richtigen". Sie erläutert das anhand von der überlappungsfreien Konturkreuzung jein < nein $\mathrm{x}$ ja, dass man nein zunächst an seiner Nachkontur -ein und seiner rhythmischen Gesamtkontur sowie das Antonym ja an seinem Anlaut leicht ablesen kann. Wenn man die Prägung *jan, in der der Tonvokal von ja übernommen und das Ausgangswort ganz enthalten ist, wird hingegen in diesem Fall weniger transparent als jein. Das hängt davon $\mathrm{ab}$, dass die Grenze zwischen den beiden Wörtern läuft mitten durch die Nachkontur: „Auf der Suche nach dem Matrixwort würde ein uneingeweihter Rezipient das Wort vermutlich eben nicht nach dem Tonvokal segmentieren (ja-n), sondern davor (j-an)“. Aus diesem Grund wird laut RonnebergerSibold ,[...] ja als eingekreuztes Wort gar nicht erkannt, weil seine beiden Phoneme nicht zusammen als bedeutungstragende Einheit betrachtet würden“" (Ronneberger-Sibold 2005: 217). Sie unterscheidet zwischen den „Konturkreuzungen“ und den

r. r. • العدد السادس والعشرون (الجزء الثالث)

(332)

مجلة كلية التربية- جامعة عين شمس 
Taghrid Ibrahim Abdelraouf Mohamed

„Halbvollkreuzungen“: In einer Konturkreuzung bleibt das eingekreuzte Wort ganz in der Kreuzung erhalten, wie z. B. Müll in Müllionärin oder kurz in Konkurz, aber das Matrixwort dagegen nicht vollständig erhalten: Das Matrixwort kann man nur an seiner rhythmischen Gesamtkontur, seinem Tonvokal und weiteren Elementen (gewöhnlich seiner Nachkontur) wahrnehmen. Unter den „Halbvollkreuzungen“" versteht Ronneberger-Sibold (2005: 218) die Kreuzungen, in denen ein Wort vollständig enthalten ist, für das andere dagegen nicht ganz erhalten wie das Matrixwort in Konturkreuzung. Diese Prägung der Halbvollkreuzungen besteht nach Ronneberger-Sibold (2005: 218) aus ,einem vollen Wort und den Resten eines zweiten (selten noch weiterer)“". Bei dem nicht voll erhaltenen Wort tritt den Mangel an die Erkennbarkeit auf, deswegen ist die struktruell-semantische Interpretierbarkeit im Sinne eines normalen Kompositums sehr wichtig für ihre Durchsichtigkeit, die dadurch unterstützt wird, wenn das voll enthaltene Wort rechts steht, „[...] wo der Rezipient bei einer versuchsweisen Interpretation als Determinativkompositum das Grundwort vermutet". Die höchste Transparenz der Halbvollkreuzungen liegen bei solchen Bildungen vor, die Konturkreuzungen maximal ähnlich sind: Ronneberger-Sibold (2005:218) bestimmt die Bedienung dafür folgendermaßen: „Das ist dann der Fall, wenn das voll erhaltene Wort die rhythmische Gesamtkontur der Kreuzung bestimmt wie ein Matrixwort, nur dass das eingekreuzte Wort nicht in es integriert ist, sondern davorgesetzt ${ }^{\prime \prime}$. Dies ist nach Ronneberger-Sibold $\mathrm{zu}$ beobachten, wenn das Vollwort mit einem Vokal beginnt, vor dem der konsonantische Anlaut des zweiten Wortes untergebracht werden kann, ohne dass die Silbenzahl erhöht wird, beispielsweise denglisch < englisch $\mathrm{x}$ deutsch oder Frice < ice $\mathrm{x}$ fresh/frisch. Das Korpus, auf das sie basiert, enthält keine Belege, bei denen die Vollform bei der Halbvollkreuzung an erster Stelle steht. Das wäre nach ihrer Behauptung geringer deutlich. Auch reduziert die Transparenz bei dem Vollwort, das an der letzten Stelle steht, wenn

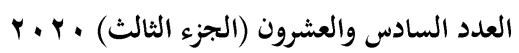

مجلة كلية التربية- جامعة عين شمس 
keines der gekreuzten Wörter die rhythmische Gesamtkontur bestimmt. In diesem Fall basiert die Durchsichtigkeit sehr stark auf die unterstützenden Faktoren wie etwa eine relativ große Usualität und Länge des gekürzten Wortes, eventuell mithilfe der Überlappung mit dem ungekürzten und von der semantischen Plausibilität der Zusammensetzung. Ronneberger-Sibold erwähnt als Beispiel die Kreuzung Austree < Australia/ Australien x tree. Zwar bleibt das wort tree vollständig in der Kreuzung vorhanden, doch legt es nicht die rhythmische Gestalt der Prägung fest, darum kann man es nicht sofort als Ausgangswort erkennen. Eine möglicherweise Segmentierung ist nach Ronneberger-Sibold z.B. Austr-ee (in Analogie zu Refer-ee), indem man versucht, die Ausgangseinheit irgendwie auf Austria oder Australien zu beziehen. Man kann die Basis erschließen, wenn man erst weiß, dass der Markenname für Teebaumöl aus Australien steht. Ganz ist schwierig die Erkennbarkeit der nicht rhythmusbewahrende Kreuzungen, bei denen das Vollwort vorangestellt ist. „Selbst wenn man weiß, dass der Name Novasil eine Metallware bezeichnet, wird man in -sil nur mit Mühe gekürztes Silber entdecken“", so erläutert Ronneberger-Sibold (2005: 219).

3. Splitterkreuzung: Der letzte Typ der Kontaminationen bezeichnet Ronneberger-Sibld (2005: 219) "Splitterkreuzung“. Darunter versteht sie, dass beide Ausgangswörter gekürzt sind, indem meistens nur Wortsplitter veschmolzen sind. Das ist der Fall bei dem häufig zitierten Beispiel Cujasuma. Die Rekonstruierbarkeit hängt dabei von der segmentalen Länge der Splitter ab und davon $\mathrm{ab}$, ob der Splitter vom Anfang oder vom Ende des gekürzten Wortes entnommen ist (vgl. Ronneberger-Sibold 2005: 220).

Hier treten etwa erst die Typen „initial - initial“ oder „,initial final“ auf, ,initial - initial“" wie z. B. Stasi < stark x sicher (Metallwaren) oder Kraba < Kramer (Firmenname) $\mathrm{x}$ Bagger (Bagger) ,initial - final“" wie z. B. Dynaject $<$ dynamisch $\mathrm{x}$ to inject . Ronneberger-Sibold (2005: 220) konstatiert, dass der zweite Typ 
seltener und transparenter ist, ,[...] vermutlich, weil er durch die Kombination eines Wortanfangs mit einem Wortende, das gewöhnlich den Tonvokal enthält, an eine Konturkreuzung erinnert". In der Tat sind die Prägungen zu sparsam, um zu feststellen, ob die höchste Transparenz wirklich auf diese distributionellen Besonderheiten zurück- zuführen ist oder nicht vielmehr auf die größere Länge der Splitter. Ronneberger-Sibold (2005: 220) behauptet, dass der eigentliche Zweck der Splitterkreuzungen ist die Bildung von opaken Neologismen aus kleinen initialen Splittern der gekreuzten Ausgangseinheiten. Ronneberger-Sibold (2005: 221) argumentiert für die Belegung der Haupttypen der Transparenzskala durch die beiden Korpora. In den literarischen Texten sind den transparentesten Typ, also die „Vollkreuzungen " bevorzugt. Bei den Markennamen beherrscht die Verwendung der mittleren Typen, also der „Konturkreuzungen“" und der „Halbvollkreuzungen“. Allerdings umfasst ein Viertel der Splitterkreuzungen Markennamen wie Cujasuma, „deren exotisches Flair durch die Opakheit begünstigt wird“. Sie fügt hinzu, dass auch die wissenschaftlichen Produkte, für Medikament und andere chemische Produkte, mit der typischen Lautgestalt lateinischer Fremdwörter den Splitterkreuzungen anordnen würden. Als Beispiel ist der Markenname Persil (Wasserstoff) Peroxyd x Natronsilikat. Zwar sind solche Bildungen nicht transparent, aber sie sind noch lang motiviert.

Ronneberger-Sibold Modell ist durch die Klarheit bei der Klassifizierung und Bezeichnung der Kontaminationsbildung gekennzeichnet. Ihre Beschreibung des Prozesses der Kontamination ist präziser und transparent als die anderen Modelle. Sinnvoll ist meines Erachtens ihre Klassifizierung der Kontamination nach der Transparenzgrad, weil der Sprachbenutzer bei der Verwendung der Kontamination in der Lage ist, verschiedene Grade der Transparenz der Kontamination zu erkennen. 


\section{Morphologische Beschreibung der Kontamination (Ālnaḥt) im Arabischen}

In diesem Kapitel werden die Formen der Kontamination nach deren Ausganswörtern von Ālsawahily (1994) und Ālbalasy (2002) durchgeführt. Dann werden die Wortklassen der Kontaminationsprodukte von Ḡirǵis (1961), Ālmağraby (1908) Ālsawahily (1994) dargestellt. Abschließend werden die morphologischen Regel bei der Bildung von Kontamination (Ālnaḥt) von Āmyn (2000) und Ḡirğis (1961) zusammengestellt.

\subsection{Formen der Kontamination im Arabischen}

Die zeitgenössischen Sprachwissenschaftler legen die morphologischen Formen der Kontaminationsbildungen fest: Die meisten gebildeten Kontaminationen werden aus vier Buchstaben geformt.

a. Wenn die Kontamination als ein transitives Verb vorkommt, wird es in Form von (f'lal) فعلل gebildet. Falls sie als ein intransitives Verb auftritt, wird es in Form von (taf'lal ) تفعلل geformt.

b. Wenn die Kontamination als einen transitiven Infinitiv auftaucht, wird er in Form von (f'lalah) فعللة gebildet und der intransitive Infinitiv wird in Form von (āltaf'lal) التفعلل geprägt.

c. Falls die Kontamination als Adjektiv vorkommt, wird es in Form von ( f'lalya) فعلائ mit der Hinzufügung der Beziehungsbuchstaben (yaā ālnasab) gebildet.

d. Wenn die Kontamination als Substantiv auftritt, soll es in einer arabischen Form gebildet werden (vgl. Ālqinīby 1992: 209, 220).

Die Kontaminationsprodukte werden nach deren Ausgangswörtern, aus denen sie geformt werden, in zwei Typen klassifiziert:

1. Kontaminationen, deren Produkte aus zwei Basiselementen gebildet werden. Als Beispiele sind: مرقسى zu امرئ القيس gehörend ist und تيم اللات zu gehörend ist (vgl. Âlsawahily 1994: 79). Dieser Typus stimmt mit dem zweiten Typus, der Ālbalasy (2002: 448) angibt, überein. Âlbalasy (2002: 448) bestimmt, dass dieser Typus 
die Kontaminationen, die aus der Verbindung der Komponenten der Genitiv- Verbindung entsteht, enthält. Als Beispiele führt Ālbalasy (2002: 448) diese Beispiele an: عبشمى، عبدرى, geformt aus: عبد شمس،.

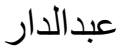

Meines Erachtens beschränken sich nicht die Kontaminationen (Ālmanhutat), die aus zwei Basiselementen entstehen, auf die Verbindung der Komponenten von Genitiv- Verbindung, sondern sie sind aus zwei Verben, zwei Substantiven oder zwei Adjektiven gebildet zu werden. Als Beispiele sind:

برق ونقش،جمع وجمد : Deformt aus: Dعمد، برقش (Giese Beispiele werden aus zwei Verben gebildet (vgl. Giirǵis 1961:69f.).

ما زهر، ماورد، فرنب gebildet aus: ماء + ورد، ماء + زهر،فار + أرنب. Diese Bildungen bestehen aus zwei Substantiven (vgl. Âlqiniby1992: 205, 239).

(vgl. Ālqiniby 1992: 205, r.A).

2. Kontaminationen, die aus mehr als drei Basiswörtern oder aus

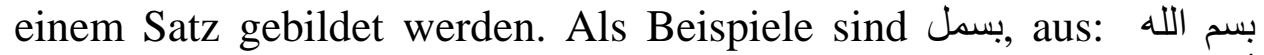
أطال الله :aus, طلبق , لا حول ولا قوة إلا بالله : aus :حوقل/حولق ,الرحمن الرحيم بقاءك الركا (vgl. Ālsawahily 1994: 80, Ālbalasy 2002: 447f., Naǵa 2008: 56,Wāfy 2004: 186, und vgl auch. Tarsy Y. . 0: 385f.).

3. Âlbalasy (2002: 447f ) fügt die Kontaminationen, die aus zwei Ausgangswörtern gebildet werden, hinzu, wobei jedes Wort eine eigne Bedeutung hat. Diese neue Bildung führt zur Sprachökonomie und neuen Bedeutung. Als Beispiel sei هل Ālbalasy konstatiert, dass solche Bildungen sehr selten in der arabischen Sprache auftreten. Es gibt keinen Unterschied zwischen die Formen der Kontamination bei den frühren und zeitgenössischen Sprachwissenschaftlern. Nur zählen sie solche Bildungen ن,

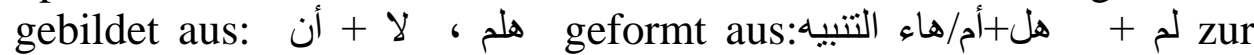
Komposition (Ālmurakab) (vgl. Ālbalasy 2002: 448). Nach den oben dargestellten Formen der Kontamination nach den Ausganggswörtern, aus denen die Kontaminationen (Ālmanhutat) gebildet werden, erkennt man, dass die Kontaminationen aus zwei

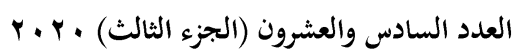

مجلة كلية التربية- جامعة عين شمس 
oder mehr als zwei Wörtern oder aus einem Satz entstehen. Man betrachtet, dass die letzte Art, deren Beispiele widersprüchlich sind, gehört nicht zur Kontamination. Es gibt viele Kontaminationsbildungen, die aus zwei Ausgangswörtern gebildet

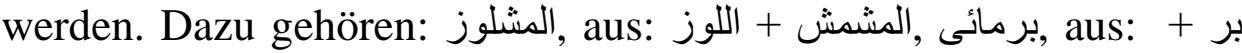
نزجنة , نزع الايدروجين u.a (vgl. Zeitschrift der Akademie für arabische Sprache 1953: 204, vgl. auch G̈irğis 1961: 75).

\subsection{Wortklasse der Kontamination( Ālnaḥt) im Arabischen}

Im Arabischen herrscht eine Übereinstimmung bei der Klassifizierung der Wortarten der Kontaminationsprodukte. Die Sprachwissenschaftler geben vier Wortklassen der Kontamination an: 1.Verbale Kontamination, 2. Adjektivische Kontamination 3. Substantivische Kontamination 4. Nisba- Kontamination:

1.Verbale Kontamination tritt im Arabischen auf, wenn ein Verb aus einem Satz geformt wird und dieses entstandene Verb bezeichnet den Inhalt oder die Artikulation der Basiswörter. Als Beispiele sind: سبحل ، دمعز، بأباً من قال سبحان الله، من قال أدام الله عزك (vgl. Ālmaǵraby 1908: 21, 'Ukašah 2002: 95, Ālsawahily 1994: 75, Tarzy 2005: 358 u. a).

2. Adjektivische Kontamination entsteht dadurch, dass aus der Verschmelzung zweier Wörter ein adjektivisches Wort gebildet wird. Beispiele für diesen Typus sind: ضبطر ، صهصلق geformt aus: ضبط +ضبر للرجل الثديد؛ صهل +صلق للثديد من الأصوات

3. Substantivische Kontamination kommt im Arabischen vor, wenn ein Substantiv aus zwei Ausgangwörtern gebildet wird, wie z. B. جلمود ، شقحطب gebildet aus: شق + حطب ، جلد + جمد .

4. "Nisba-Kontamination" ist im Arabischen vorhanden, wenn ein Wort, die aus zwei Wörtern oder aus den Komponenten der GenitivVerbindung in Beziehung zu einem Land oder einer Person steht, gebildet wird. Als Beispiele geben die Sprachwissenschaftler diese Bildungen an:

$$
\text { طبرستان + خوارزم، عبد+ : gebildet aus ، حنفلتى ، شفعنى، طبرخزى ، عنشمى +حنيفة }
$$$$
\text { العدد السادس والعشرون (الجزء الثالث) •r.r }
$$ 
حنيفى + (vgl. Ālmaǵraby 1908: 21f., 'Ukašah 2002: 95f., Ālsawahily 1994: 75-79 Tarzy 2005: 357f. u. a). Ḡirgis (1961: 65) stellt die morphologische Beschreibung der Wortklassen von den Kontaminationsformen (Āl manhutat) zusammen. Seine Darstellung fügt weitere Erklärung der Kontaminationsformen hinzu, wobei man nicht nur die Wortklassen erkennt, sondern man versteht auch die morphologische Beschreibung der gebildeten Kontaminationen.

Er weist darauf hin, dass aus den meisten gebildeten Kontaminationsprodukten ein Verb und einen Infinitiv geformt werden. Als Beispiele sind: حمدل يحمدل حمدلة ، بسمل يبسمل بسملة

(بعثر يبعثر، بعثرة

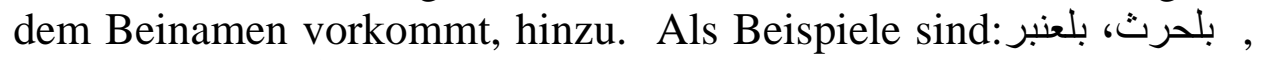
gebildet aus: بنى الحارث، بنى العنبر.

Bei diesen Bildungen wird (Ālnun) für die Erleichterung getilgt. Die Darstellung der morphologischen Beschreibungen nach G̈irgis (1961: 63-65) wird im Folgenden ausführlich angeführt.

\section{3 Morphologische Regel bei der Bildung von Kontamination}

Es gibt keine bestimmte Regel bei der Bildung von Kontaminationsformen in den traditionellen und modernen Fachbüchern, aber die meisten Sprachwissenschaftler weisen auf die zwei wichtigsten Voraussetzungen bei der Bildung von Kontamination hin:

1. Man soll bei der Bildung von Kontamination die bekannten arabischen Formen berücksichtigen: Die arabischen Formen der 3. radikalen, 4. radikalen und 5. radikalen Wurzeln.

2. Die Buchstaben der neuen gebildeten Wörter sollen harmonisch klingen (vgl. Āmyn 2000: 431, Ḡirǵis1961: 63 u. a.). G̈irğis (1961: 63 -65) stellt im Hinblick auf die gebildeten Kontaminationsformen bestimmte morphologische Regel zusammen:

Erstens: Man kann Kontamination aus allen Ausgangswörtern oder aus einigen Ausgangswörtern bilden:

1. Die Kontamination wird nur durch das erste Ausgangswort geformt, wenn das erste Wort vier oder mehr als vier

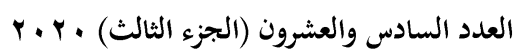

(339)

مجلة كلية التربية- جامعة عين شمس 
Buchstaben enthält. In diesem Fall wird (f'lal) فعل durch das erste Wort ohne das Zweite gebildet: حيهل besteht aus: حيهلا بالثئ

Die Kontaminationsformen werden aus zwei oder mehr als zwei Ausgangswörtern gebildet:

a. Kontamination aus zwei Ausgangswörtern: جعلت : جعل : جeformt aus فـالك.

b. Kontamination aus drei Ausgangswörtern: دمعز geformt aus: أدام الله عز5.

c. Kontamination aus vier Ausgangswörtern: بسم الله . بسمل

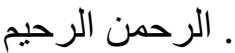

d. Kontamination aus sechs Ausgangswörtern: حولق أو حوقل geformt aus:

Gēirǵis vernachlässigt hier bei der Darstellung der Kontaminationsformen die Bildung der Kontamination aus fünf Ausgangswörtern. Er konstatiert, dass die Sprachwissenschaftler auf das Wort (الله) bei der Verschmelzung der Ausgangswörtern verzichten (vgl. Girǵis 1961: 63f.).

2.Wenn die Kontamination aus zwei Ausgangswörtern gebildet wird, dessen erstes Wort drei Buchstaben hat, wird das ganze erste Ausgangswort in den meisten Fällen in der Bildung vorkommen und (f'lal) فعلل wird durch das Zweite ergänzt: ويلمه، برق وبرو geformt aus: ويل+ أمه برق+ قال فيل Ausnahme sind nach G̈irǵis ( 1961: 64) diese Beispiele: سمعل، جعفد aus: السلام عليكم جعلت فداكل. Bei diesen Beispielen werden zwei Buchstaben aus dem ersten und zweiten Ausgangswort genommen. Er behauptet, dass die Sprachwissenschaftler nicht vorausgesetzt haben, dass das ganze Ausgangswort in der Bildung vorhanden sein muss.

3.Wenn das erste Ausgangswort mit dem bestimmten Artikel ( $\bar{A} 1)$ anfängt, wird diesr Artikel getilgt und die Kontamination durch die obengenannten Regel gebildet: حمدل geformt aus: الحمد لله.

4.Wenn das erste Ausgangswort mit (Í، Ú ‘Á) (hurwf āl 'ylah) endet, wird der letzte Buchstabe (lām Ālkalimh) getilgt und فعل (f'lal)

r. r. العدد السادس والعشرون (الجزء الثالث)

$(340)$

مجلة كلية التربية- جامعة عين شمس 
Taghrid Ibrahim Abdelraouf Mohamed

wird durch das zweite Ausgangswort ergänzt: بكلف geformt aus: بلا كيف.

5. Wenn das erste Ausgangswort mit dem ersten Personalpronomen ( ضمير المتكلم المتصل) (damīr Āl mutakalim Ālmutșil) endet, wird es getilgt: حسبل geprägt aus: حسبى الله

6.Bei der Bildung der Kontamination aus zwei Ausgangswörtern, dessen erstes Ausgangswort mehr als drei Buchstaben enthält, wird eine von den drei Weisen befolgt:

a. Zwei Buchstaben aus dem ersten und dem zweiten Ausgangswort werden in der Bildung verschmolzen: سمعل geformt aus: السلام عليكم.

b. Bei der Prägung der Kontamination werden die zusätzlichen Buchstaben aus dem ersten Ausgangswort getilgt und ein Verb wird dadurch gebildet. Außerdem wird فعلل (f'lal) durch das zweite Ausgangswort ergänzt: سبحل aus: سبحان الله .

c. Die Form فعلل (f'lal) wird dadurch gebildet, dass das erste Ausgangswort ohne das zweite Ausgangswort in der Bildung tritt: حيهل geformt aus: حيهلا بالثئ

Zweitens: Die Reihenfolgen der ursprünglichen Buchstaben der Ausgangswörter werden bei der Bildung der Kontamination berücksichtigt. Ḡirgis ( 1961: 64) behauptet, dass die Bildungen, die dieser Regel nicht befolgt werden, sind ausgenommen: طبلق، حوقل : طلنق، حولق : geformt aus:

أطال الله بقاءك، لا حول و لا قوة إلا بالله (vgl. G̈irǵis 1961: 64).

Drittens: Die Berücksichtigung der ursprünglichen Konsonanten und Vokalen bei der Prägung der Kontamination sind nicht vorausgesetzt.

Viertens: Die Form فعلل (f'lal) wird durch die Wiederholung des ersten Teiles des ersten Ausgangswortes gebildet: بأباً aus: بأبى أنت (vgl. Girǵgis 1961: 63-65). Die oben dargestellten Regel werden von einigen zeitgenössischen Sprachwissenschaftlern abgelehnt und von anderen akzeptiert ( vgl. Girğis 1961: 77f.). Aus den oben dargestellten Regeln erkennt man, dass Girǵis die morphologishe Beschreibung der Kontaminationsformen im Hinblick auf einige

r. r. . العدد السادس والعشرون (الجزء الثالث)

(341)

مجلة كلية التربية- جامعة عين شمس 
beschränkte Beispiele durchführt, infolgedessen gibt es kein bestimmtes Modell, das man bei der Bildung von Kontaminationen (Ālmanhutat) benutzen kann. Ich bin der Meinung, dass die Festlegung bestimmter Regel bei der Bildung von Kontamination nicht mit allen Kontaminationsbildungen entsprechen, wobei man einige Bildungen insbesondere im Bereich der Wissenschaften betrachtet, die nicht diesen Regeln befolgen. Dafür sind Beispiele wie: كلر، لأمن، لبرم, aus: نزع الأمين، نزع البروم، نزع الكلور. Bei diesen Bildung tritt nicht das Wort نز لنز Kontaminationen auf, trotzdem hält die Akademie für Arabische Sprache sie für Kontaminationen (vgl. Zeitschrift der Akademie für Arabische Sprache 1953/7: 204).

\section{4 Schlussfolgerungen:}

Die Kontamination ist ein selbsständiger Wortbildungstyp, der in den beiden Sprachen vorhanden ist. Und jede Sprache hat ihre morphologischen Besonderheiten und ihre bestimmte Regel bei der Bildung von Kontamination. Die Kontaminationen kommen als Substantive, Adjektive, Verben in den beiden Sprachen vor. Im Deutschen können fast alle Wortarten als Kontamination auftreten. Im Arabischen taucht die Nisba- Kontamination auf. In den beiden Sprachen entsteht die Kontamination entweder durch die Verschmelzung mindestens zweier Wörter zu einem Wort oder durch die Überlappung der Ausgangswörter und durch diese Überlappung wird ein neues Lexem gebildet. Im Deutschen sind die Kontamination bei Substantiven produktiv, seltener bei Adjektiven und Verben. Im Arabischen ist dieses Phänomen dagegen bei Verben und Adjektiven produktiv. Die Kontamination hat im Deutschen zwei Haupttypen: Wortkreuzung und Wortüberschneidung. 


\section{Literaturverzeichnis:}

\section{Deutsche Literatur}

Bußmann, Hadumod (Hg.) (2002): Lexikon der Sprachwissenschaft. 3., aktualisierte und erweiterte Aufl. Stuttgart. S. 373-373.

Donalies, Elke (2011): Basiswissen Deutsche Wortbildung. 2. Übarb.Aufl. Stuttgart/ München, Wien.

Friedrich, Cornelia (2008): Kontamination - Zur Form und Funktion eines Wortbildungstyps im Deutschen. Dissertation Universität Erlangen-Nürnberg.

Kessel, Katja/Reimann, Sandra (2008): Basiswissen. Deutsche Gegenwartssprache. 2.,überarb. Aufl. Tübingen.

Lohde, Michael (2006): Wortbildung des modernen Deutschen: Ein Lehrund Übungsbuch. Tübingen.

Ronneberger-Sibold, Elke (2005): Zur Definition und Typologie von Wortkreuzungen. Ein Vorschlag auf der Grundlage ihrer relativen Transparenz. In: Sprache und Natürlichkeit. Gedenkband für Willi Mayerthaler. Hrsg. v. Gertraud Fenk-Oczlon und Christian Winkler. Tübingen, 205-224.

\section{Arabische Literatur}

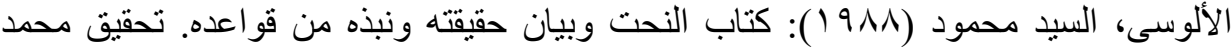

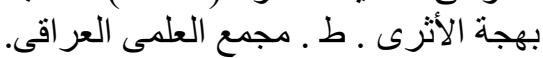

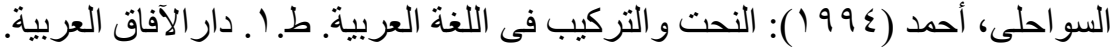

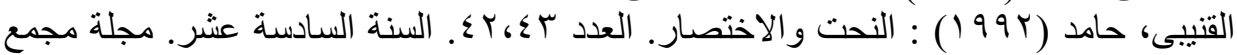
اللغة العربية الاردنى.

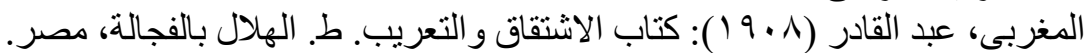

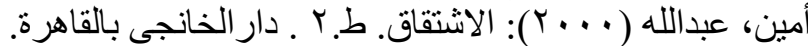

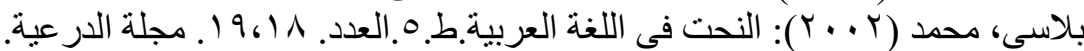

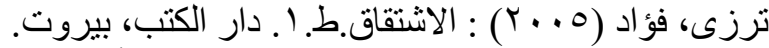

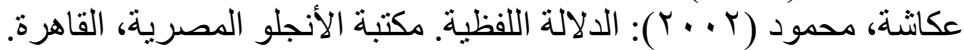

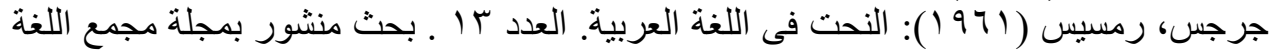

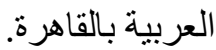

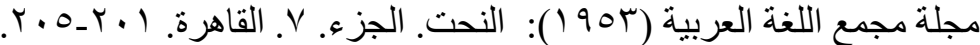

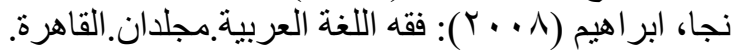

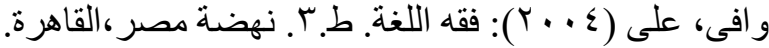

العدد السادس والعشرون (الجزء الثالث) •r.r 